\title{
OPEN Intrinsic signal optoretinography of dark adaptation kinetics
}

\author{
Tae-Hoon Kim ${ }^{1}$, Jie Ding ${ }^{1} \&$ Xincheng Yao ${ }^{1,2} \bowtie$ \\ Delayed dark adaptation due to impaired rod photoreceptor homeostasis has been reported as the \\ earliest symptom of eye diseases such as age-related macular degeneration, diabetic retinopathy, \\ and retinitis pigmentosa. Objective measurement of dark adaptation can facilitate early diagnosis \\ to enable prompt intervention to prevent vision loss. However, there is a lack of noninvasive \\ methods capable of spatiotemporal monitoring of photoreceptor changes during dark adaptation. \\ Here we demonstrate functional optical coherence tomography $(O C T)$ for in vivo intrinsic signal \\ optoretinography (ORG) of dark adaptation kinetics in the C57BL/6J mouse retina. Functional OCT \\ revealed a shortening of the outer retina, a rearrangement of the cone and rod photoreceptor \\ interdigitation zone, and a reduction in intrinsic signal amplitude at the photoreceptor inner segment \\ ellipsoid (ISe). A strong positive correlation between the outer retinal shortening and ISe intensity \\ reduction was also confirmed. Functional OCT of dark adaptation kinetics promises an objective \\ method for rapid ORG assessment of physiological integrity of retinal photoreceptors.
}

Dark adaptation refers to a systematic recovery of visual sensitivity in the dark following exposure to bright lights ${ }^{1}$. During dark adaptation, multiple processes simultaneously occur to maintain retinal homeostasis, including photoreceptor repolarization ${ }^{2}$, photopigment regeneration ${ }^{2}$, blood flow modulation ${ }^{3}$, a redistribution of photoreceptor signaling protein $s^{4}$ and interphotoreceptor matrix proteins $s^{5}$, and a reversal of metabolic energy flow ${ }^{6}$. Visual perception in night vision is rod-mediated; thus, delayed dark adaptation due to impaired rod photoreceptor homeostasis is often the earliest symptom of various retinal diseases, such as age-related macular degeneration $(\mathrm{AMD})^{7}$, diabetic retinopathy $(\mathrm{DR})^{8}$, and retinitis pigmentosa $(\mathrm{RP})^{9}$, the leading causes of irreversible vision loss. Since early detection is essential to prevent further vision loss ${ }^{10-13}$, the measurement of dark adaptation kinetics has been proposed as a potential solution for screening early-stage retinal diseases.

Conventional dark adaptation measurement is psychophysical, i.e., dark adaptometry or electrophysiological, i.e., electroretinogram $(E R G)^{7,14}$. While providing quantitative information on retinal dysfunction, these methods have a limited spatial resolution. Considering the coherent interactions between retinal cells, it appears important to quantitatively examine functional activity simultaneously as well as anatomical arrangement.

Recent developments of intrinsic signal optoretinography (ORG) promise a new methodology for the noninvasive objective assessment of dark adaptation. ORG generally refers to functional optical imaging of the retina. Functional optical coherence tomography (OCT) promises a depth-resolved imaging modality to enable intrinsic signal ORG of the retina ${ }^{15-26}$. Functional OCT has revealed intrinsic optical signal (IOS) distortions correlated with pathophysiological conditions in a mouse model of $\mathrm{RP}^{27}$ and human RP subjects ${ }^{20}$. Previous OCT studies of the dark-adapted mouse retina revealed outer retinal shortening along with a reduction in the width of a hypo-reflective band between the photoreceptor outer segments (OS) and retinal pigment epithelium (RPE) ${ }^{28-30}$. Lu et al. ${ }^{31}$ also observed photoreceptor OS elongation following strong light exposure and subsequent recovery, i.e., photoreceptor OS shortening, during dark adaptation in human subjects. However, dynamic monitoring of transient structural and reflectivity changes, which promises a biomarker for rapid assessment of functional integrity of dark adaptation in the retina, remains to be explored.

Here we report intrinsic signal ORG assessment of dark adaptation kinetics in the mouse retina in vivo. The light-adapted retina was initially measured as a baseline, followed by subsequent recording under dark conditions. Dark-induced structural and reflectivity changes in the outer retina were clearly observed in OCT imaging. High-speed monitoring further identified a strong correlation between structural and physiological changes in the outer retina. This study demonstrates the potential of intrinsic signal ORG assessment of dark adaptation kinetics in the living retina.

${ }^{1}$ Department of Biomedical Engineering, University of Illinois at Chicago, Chicago, IL 60607, USA. ${ }^{2}$ Department

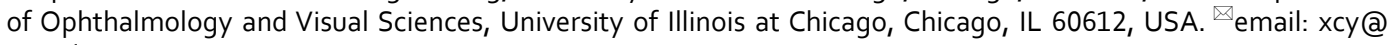
vic.edu 


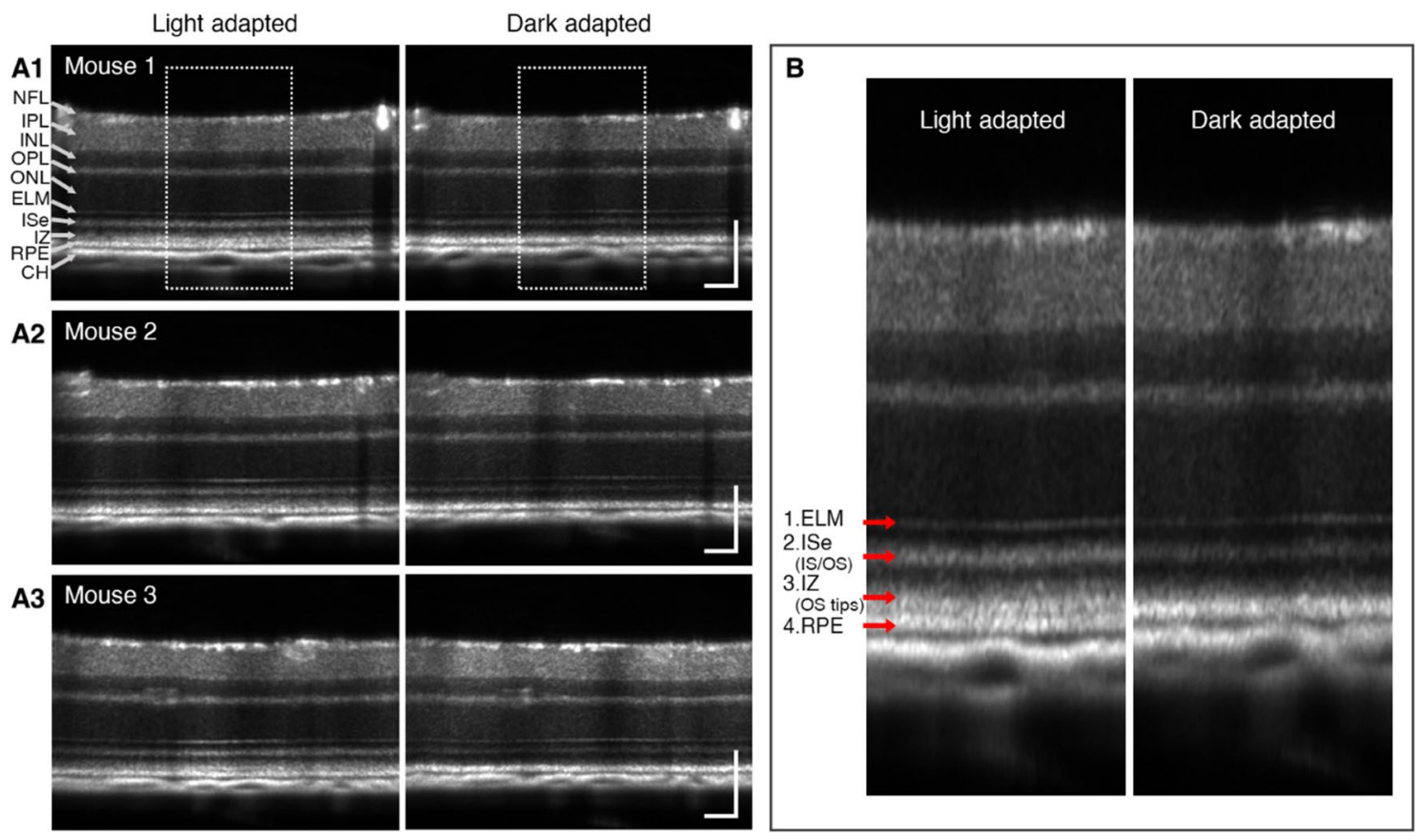

Figure 1. Comparison of light- and dark-adapted retinas. (A) OCT images of light- and dark-adapted retinas from 3 different mice (A1-A3). (B) Enlarged view of the white rectangle regions in (A1). Red arrows indicate OCT hyper-reflective bands in the outer retina. NFL nerve fiber layer, IPL inner plexiform layer, INL inner nuclear layer, $O P L$ outer plexiform layer, $O N L$ outer nuclear layer, ELM external limiting membrane, ISe inner segment ellipsoid, $I Z$ interdigitation zone, $R P E$ retinal pigment epithelium, $C H$ choroid, $I S$ inner segment, $O S$ outer segment. Scale bars: $100 \mu \mathrm{m}$.

\section{Results}

Imaging features of light- and dark-adapted mouse retinas. Adult C57BL/6 J mice were used in this study. The experiment was performed during the early afternoon when the mouse retina was light-adapted by constant ambient light conditions. Total seven retinal OCT volumes were sequentially acquired at the same region on the dorsal quadrant per mouse; 1st OCT for light-adapted baseline measurement and 2nd-7th OCTs for dark adaptation measurement, acquired at every $5 \mathrm{~min}$ interval for $30 \mathrm{~min}$. To first validate the adaptation effects on the retina, a direct comparison between the light-adapted (1st OCT) and dark-adapted (7th OCT) retinas was performed. Figure 1A shows representative OCT images from different mouse retinas. We noted three distinct features in the retina under different light conditions (Fig. 1B). First, it was readily recognizable that dark adaptation caused a reduction in outer retinal thickness. Second, in the light-adapted retina, the interdigitation zone (IZ) and OS tip band of the outer retina (the 3rd outer retinal band) was distinguished, which was absent in the dark-adapted retina, and a hypo-reflective band between the IZ and RPE band was only observed in the light-adapted retina. Third, OCT intensity in the inner segment ellipsoid (ISe) and IS/OS band (the 2nd outer retinal band) markedly decreased in the dark-adapted retina. These results demonstrate that dark adaptation induces transient structural and reflectivity changes in the retina.

Quantitative analysis of retinal changes during dark adaptation. Figure 2A shows repeated measures of OCT during $30 \mathrm{~min}$ dark adaptation. To quantify retinal changes, average OCT A-lines were prepared and used for retinal thickness comparison at different time points. We found that the inner retina showed little change (Fig. 2B), while the outer retina revealed a significant reduction in thickness during dark adaptation (Fig. 2C). A repeated-measures analysis of variance (ANOVA) determined that mean outer retinal thickness differed significantly between different time points $(p<0.001)$. Post-hoc tests using the Bonferroni correction revealed that even $5 \mathrm{~min}$ dark adaptation resulted in a significant reduction in outer retinal thickness (Light: $106.8 \pm 2 \mu \mathrm{m}$; 5 min dark: $105.1 \pm 1.9 \mu \mathrm{m} ; p<0.001)$. However, dark adaptation had not significantly altered inner retinal thickness (Light: $79.42 \pm 1.7 \mu \mathrm{m} ; 30$ min dark: $78.65 \pm 2 \mu \mathrm{m} ; p=0.15$ ). Next, the outer retina was segmented into two regions and analyzed (Fig. 2D,E). A slight decline was observed in the outer nuclear layer (ONL) thickness (Fig. 2D). In contrast, the external limiting membrane (ELM)-RPE complex revealed a rapid decline in thickness right after the light went off. A repeated-measures ANOVA determined that mean ONL $(p<0.001)$ and ELM-RPE $(p<0.001)$ thickness differed significantly between the time points, and post-hoc tests using the Bonferroni correction revealed that statistically significant changes were first observed after 20 min dark adaptation in the ONL (Light: $58.08 \pm 1.6 \mu \mathrm{m} ; 20$ min dark: $56.76 \pm 1.5 \mu \mathrm{m} ; p<0.01$ ) and 5 min dark adaptation in the ELM-RPE complex (Light: $48.73 \pm 1.6 \mu \mathrm{m} ; 5$ min dark: $47.08 \pm 1.4 \mu \mathrm{m} ; p<0.001$ ). These results are in line with previous observations ${ }^{29,30}$ and demonstrate that ELM-RPE complex predominantly affects retinal thin- 

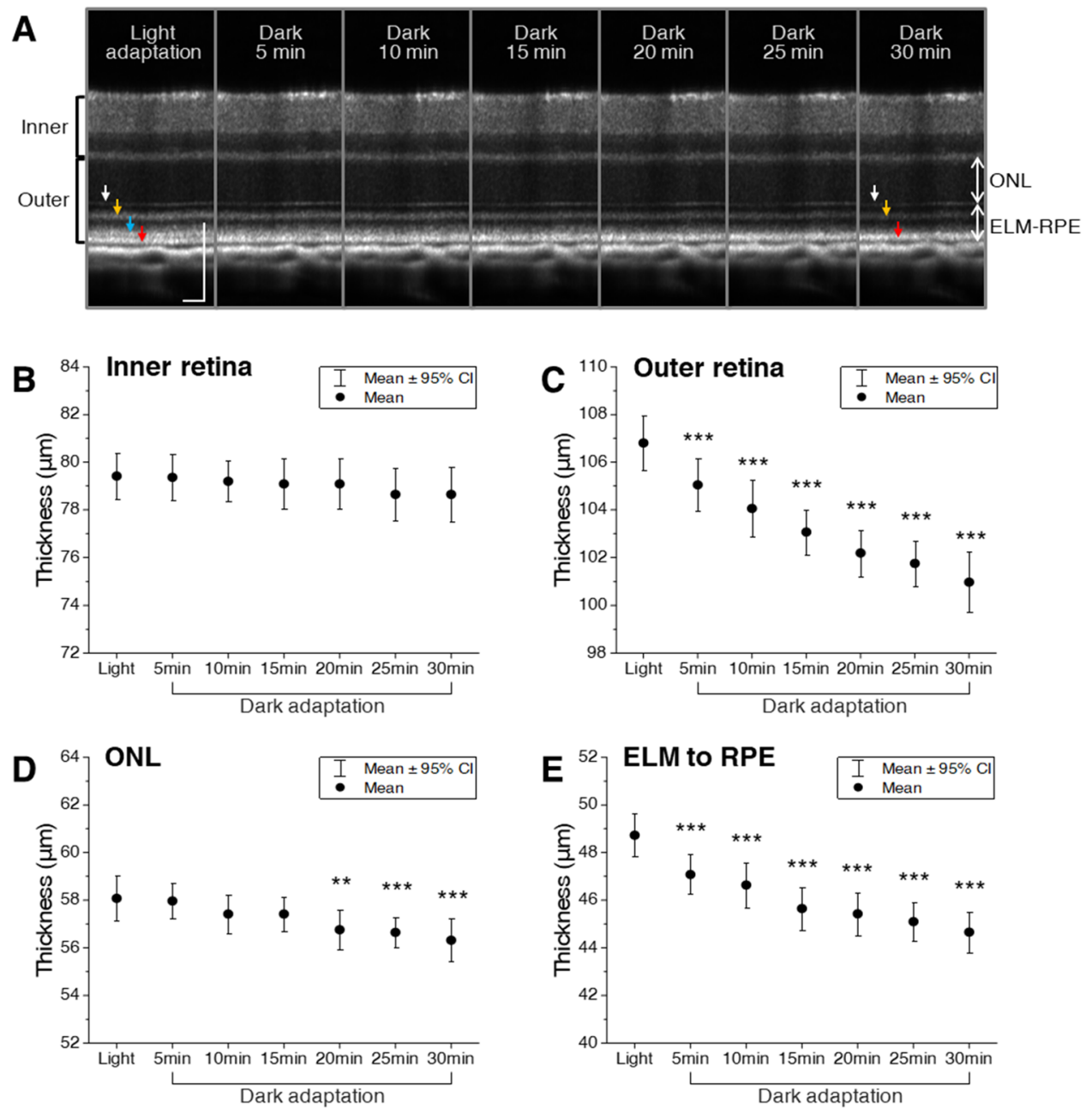

Figure 2. Quantitative measurement of retinal thickness changes during dark adaptation. (A) A sequence of OCT images was obtained every $5 \mathrm{~min}$ up to $30 \mathrm{~min}$ during dark adaptation. Arrows to indicate outer retinal bands: 1st ELM band (white), 2nd ISe and IS/OS band (yellow), 3rd IZ and OS tip band (blue), 4th RPE band (red). ONL outer nuclear layer, ELM external limiting membrane, RPE retinal pigment epithelium, ISe inner segment ellipsoid, IS inner segment, OS outer segment. Thickness measurement of $(\mathbf{B})$ the inner retina, $(\mathbf{C})$ outer retina, (D) ONL, and (E) ELM-RPE complex during dark adaptation. Data are represented as mean with $95 \%$ confidence intervals (error bars). Significant differences between time points were tested using a one-way repeated measures ANOVA with Bonferroni's post-hoc tests. $\mathrm{N}=14 .{ }^{*} p<0.05,{ }^{* *} p<0.01,{ }^{* *} p<0.001$ compared to the light-adapted retina. Scale bars: $100 \mu \mathrm{m}$.

ning, accounting for $\sim 70 \%$ reduction of the outer retina (ELM-RPE displacement after 30 min dark: $-4.07 \mu \mathrm{m}$; total outer retinal displacement after 30 min dark: $-5.83 \mu \mathrm{m})$.

The OCT signal is highly sensitive to changes of intrinsic optical properties in excitable cells, such as light scattering, polarization, and absorption fluctuation $\mathrm{s}^{32,33}$. As the OCT intensity reduction was apparent in the ISe band (Fig. 1B), we further analyzed relative intensity changes in different hyper-reflective bands, including inner plexiform layer (IPL), outer plexiform layer (OPL), ISe, and RPE. The average OCT A-lines were used for the relative intensity measurement after intensity normalization based on the ONL reflectance. Figure 3 showed that the ISe intensity had significantly reduced after 5 min dark adaptation $(p<0.001)$. Relative to the baseline measurement, the IPL, OPL, and RPE also showed a significant intensity reduction but at the later phase of dark adaptation compared to the ISe change (post-hoc tests using the Bonferroni correction: IPL at $20 \mathrm{~min}(p<0.01)$; OPL at $15 \mathrm{~min}(p<0.01)$; RPE at $20 \mathrm{~min}(p<0.05))$.

As a control experiment, we also tested potential anesthesia effects on intrinsic signal changes (Fig. 4). The ketamine-xylazine mixture is known to cause cardiodepressive effects that lower local blood flow and vessel diameter over time ${ }^{34,35}$. Since the effect is time-dependent, it is necessary to carefully manage the imaging time point from the anesthesia. Seven retinal OCT volumes were sequentially acquired over 30 min under ambient light conditions, and it took 8-10 min between the time of intraperitoneal injection and the first OCT volume acquisition. The results showed that there was no significant change in both ISe band intensity and outer retinal thickness (Fig. 4B,C). Collectively, dark adaptation induces significant IOS changes as well as outer retinal thinning. 


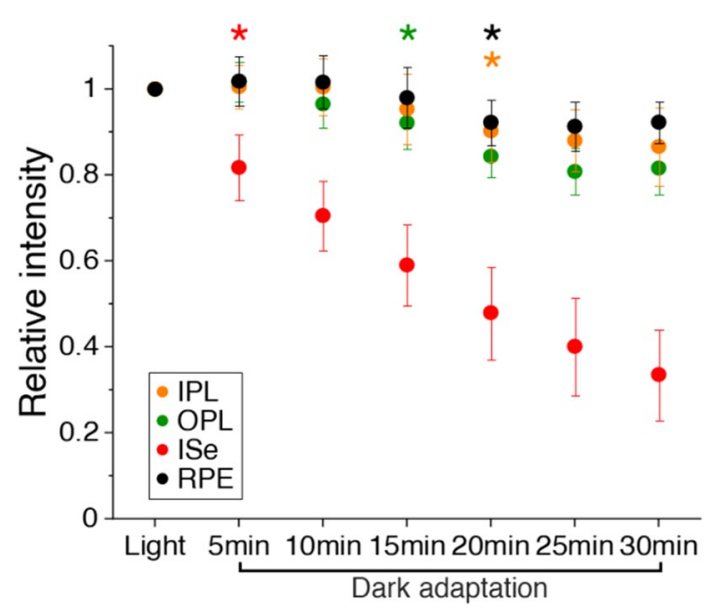

Figure 3. Relative OCT intensity measurement of hyper-reflective bands in the retina. Data are represented as mean with $95 \%$ confidence intervals (error bars). Significant differences between time points were tested using a one-way repeated measures ANOVA with Bonferroni's post-hoc tests. $\mathrm{N}=14$. Asterisks indicate the earliest time point showing a statistically significant difference $\left({ }^{*} p<0.05\right)$ in each band compared to the baseline lightadapted measurement.
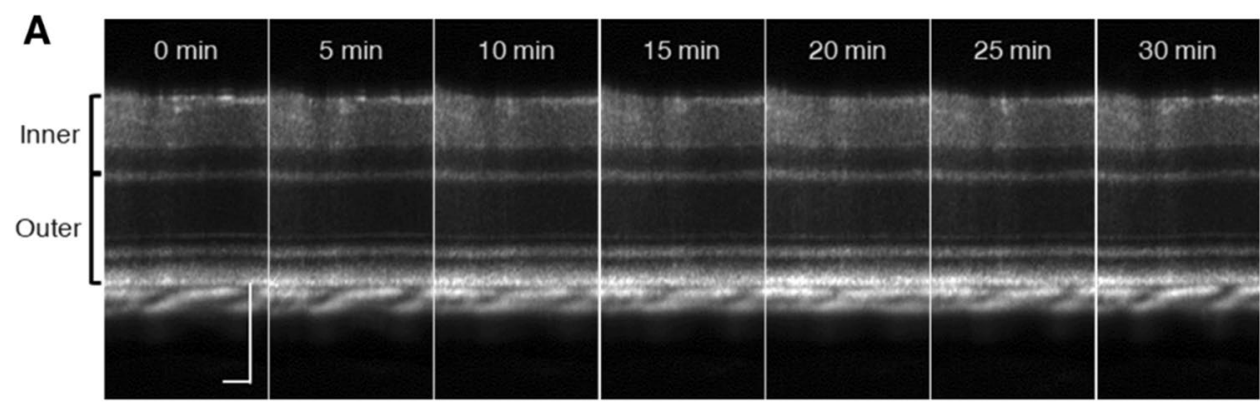

B

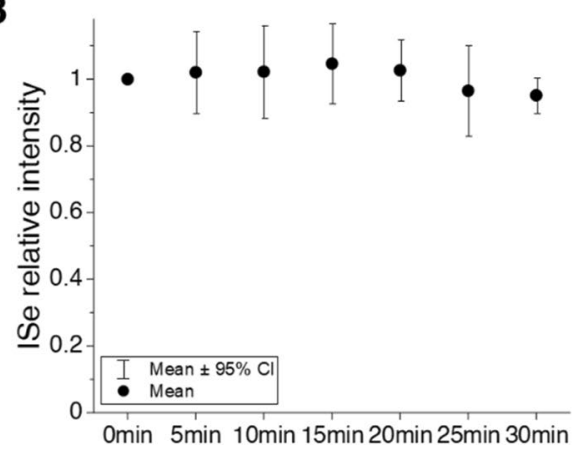

C

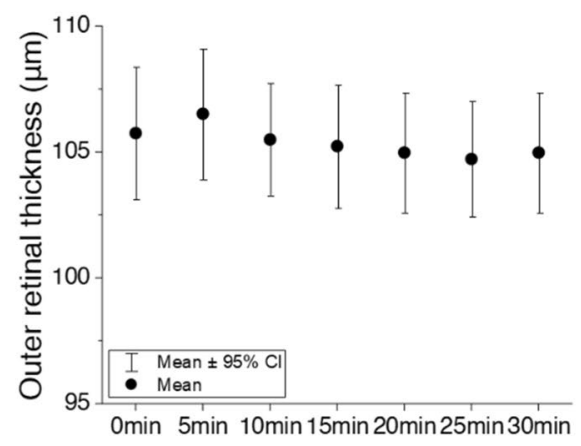

Figure 4. (A) Sequential OCT images obtained under ambient light conditions. (B) Relative OCT intensity measurement of the ISe band and $(\mathbf{C})$ outer retinal thickness measurement under ambient light conditions. Data are represented as mean with $95 \%$ confidence intervals (error bars). $\mathrm{N}=6$. Scale bars: $100 \mu \mathrm{m}$.

High-speed monitoring of a light-dark transitional period. Notable retinal changes were observed even after 5 min dark adaptation (Figs. 2 and 3), likely suggesting that OCT may detect early changes of the retina during a short transitional period to darkness. High-speed B-scan recordings were thus performed with $62.5 \mathrm{~ms}$ temporal resolution at a fixed retinal plane. In a total $5 \mathrm{~min}$ recording, the light-adapted retina as a baseline was imaged for the first $30 \mathrm{~s}$, and the retina was continuously being imaged for $4.5 \mathrm{~min}$ in darkness. The lights were off at $30 \mathrm{~s}$. Figure 5A exhibits a motion scan (M-scan) illustrating spatiotemporal relations, where each column represents an average A-scan, and individual A-scans were vertically aligned based on the ELM's peak location. Figure 5B shows the vertically enlarged view of the outer retina in Fig. 5A. In line with the previous results (Figs. 1 and 2), it was observed that the 3 rd outer retinal band (i.e., IZ and OS tips) became elusive 

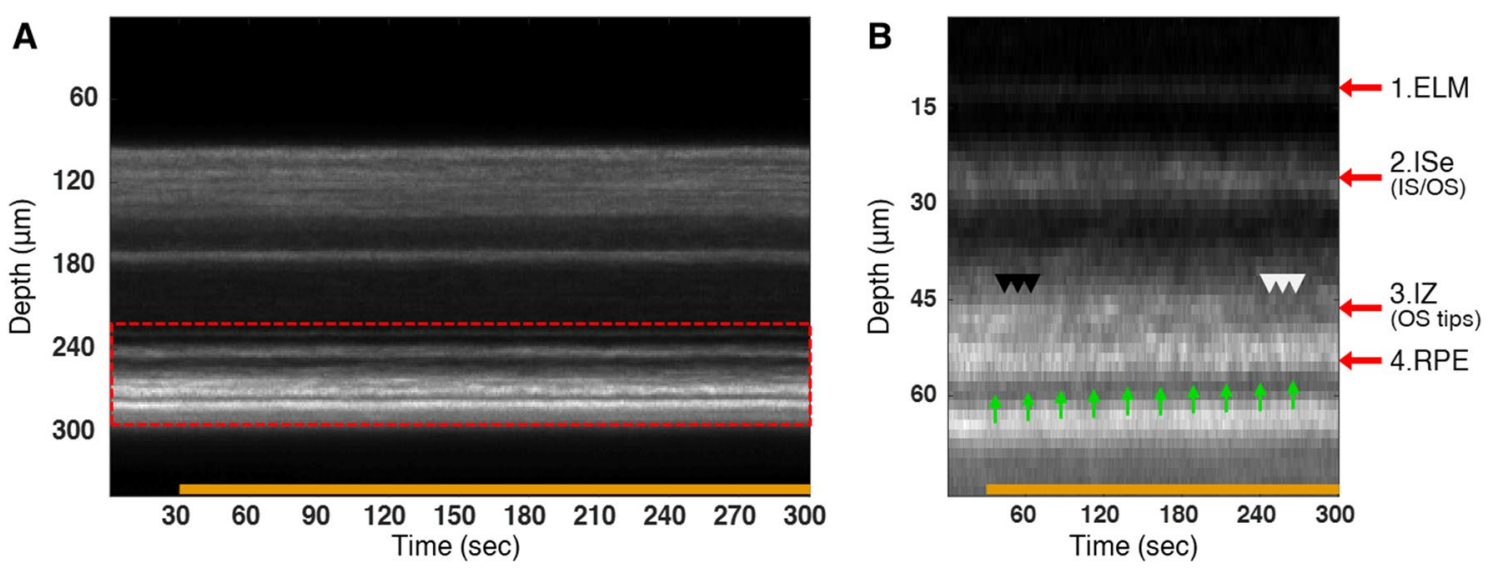

Figure 5. High-speed OCT recording during the light-dark transition. The lights were off at $30 \mathrm{~s}$. (A) Spatiotemporal mapping of OCT images. Each column represents a 1-s recording. (B) Enlarged view of the red box in (A). Black and white arrowheads point out the 3rd band change during dark adaptation. Green arrows indicate basal RPE position. ELM external limiting membrane, ISe inner segment ellipsoid, IS inner segment, OS outer segment, $I Z$ interdigitation zone, $R P E$ retinal pigment epithelium, $\mathrm{CH}$ Choroid.

over time (white arrowheads in Fig. 5B), and the boundary between the RPE and choroid shifted toward the inner retinal side (green arrows in Fig. 5B), indicating the outer retina became short.

Further detailed analysis was made by color-coded intensity profiles (Fig. 6A). Different colors represent different time points; dark red and blue indicate the early and late phases of recording, respectively. From the results, we found three imaging features. First, ISe intensity reduction rapidly occurred after lights off (Fig. 6B). Second, as seen in Fig. 5B, outer retinal bands, including the RPE and choroid, were being shifted toward the ELM side (Fig. 6C). These observations were consistent with the results presented in Figs. 2 and 3. Third, the colorcoded intensity profiles delineate that the $3 \mathrm{rd}$ band decreased in contrast over time (Fig. 6D). Intriguingly, we observed not only a peak at the OS tip position (black arrows in Fig. 6A,D) but also another peak in the midway of the OS (green arrows in Fig. 6A,D), and both peaks tended to gradually decrease in intensity right after lights off. In addition, a linear trend between retinal responses and time courses of dark adaptation was estimated by Pearson correlation tests (Fig. 6E,F). Results showed that dark adaptation time was negatively correlated with ISe intensity $(r=-0.933)$ and ELM-RPE displacement $(r=-0.982)$. We also found a strong positive relationship between ISe intensity and ELM-RPE displacement $(\mathrm{r}=+0.944)$ (Fig. 6G). Collectively, these data suggest that retinal changes gradually occur in a linear manner upon darkness, and high-speed spatiotemporal mapping enables the measurement of prompt retinal activities due to dark adaptation.

Double OS peaks may represent cone and rod tips. An interesting observation in this study was a moderate-reflective peak above the conventional hyper-reflective 3rd band (i.e., rod IZ and OS tips) (Fig. 6A,D), suggesting the presence of an additional 3rd outer retinal band. Since this additional peak was consistently observed in 13 out of 14 mice, it was less likely an artifact. The first 3rd band (i.e., the ISe side, green arrows in Fig. 6A) was relatively gentle compared to the second 3rd band (i.e., the RPE side, black arrows in Fig. 6A), and both peaks gradually decreased in intensity during dark adaptation (Fig. 6A,D). A peak-to-peak distance was measured from the ISe to each 3rd band peak, and we found that the distances were $13.4 \pm 1.8 \mu \mathrm{m}$ for the first peak and $20.9 \pm 2.2 \mu \mathrm{m}$ for the second peak (Fig. 7A), well matching the cone and rod OS length in the mouse retina $^{36}$. A representative $\mathrm{M}$-scan image by averaging 14 different $\mathrm{M}$-scans was also constructed to reveal a layered intensity distribution (Fig. 7B1). The distribution was not a continuous gradient along the depth direction; it was rather a discrete staircase distribution (Fig. 7B2). This pattern became more obvious when OCT intensity was depicted with a color-lookup-table (Fig. 7C). Collectively, our results demonstrate that the 3rd outer retinal bands in the mouse retina can be divided in two parts: the first moderate-reflective band and second hyperreflective band.

\section{Discussion}

In this study, we demonstrated ORG measurement of dark adaptation in the mouse retina. Functional OCT as an ORG modality was sequentially collected before and after lights off to investigate retinal status under different light conditions. We found that retinal response during dark adaptation was reflected by transient structural (i.e., retinal thickness and OCT bands integrity) and physiological (i.e., intrinsic optical signals) changes, mainly in the outer retina. These responses were manifested as a function of the adaptation time and readily detectable upon darkness. We assumed that coherent interactions between the photoreceptors and RPE to be adapted in the absence of light may be directly or indirectly reflected in OCT imaging.

The altered reflectance observed in the ISe band can support the notion that metabolic states of the photoreceptors can be associated with intrinsic signal changes in the ISe ${ }^{37,38}$. The photoreceptors display the highest rate of oxidative metabolism in the body and regulate a flow of metabolic energy differentially in light and dark conditions. Energy demands and oxygen consumption are more significant in darkness than in light due 

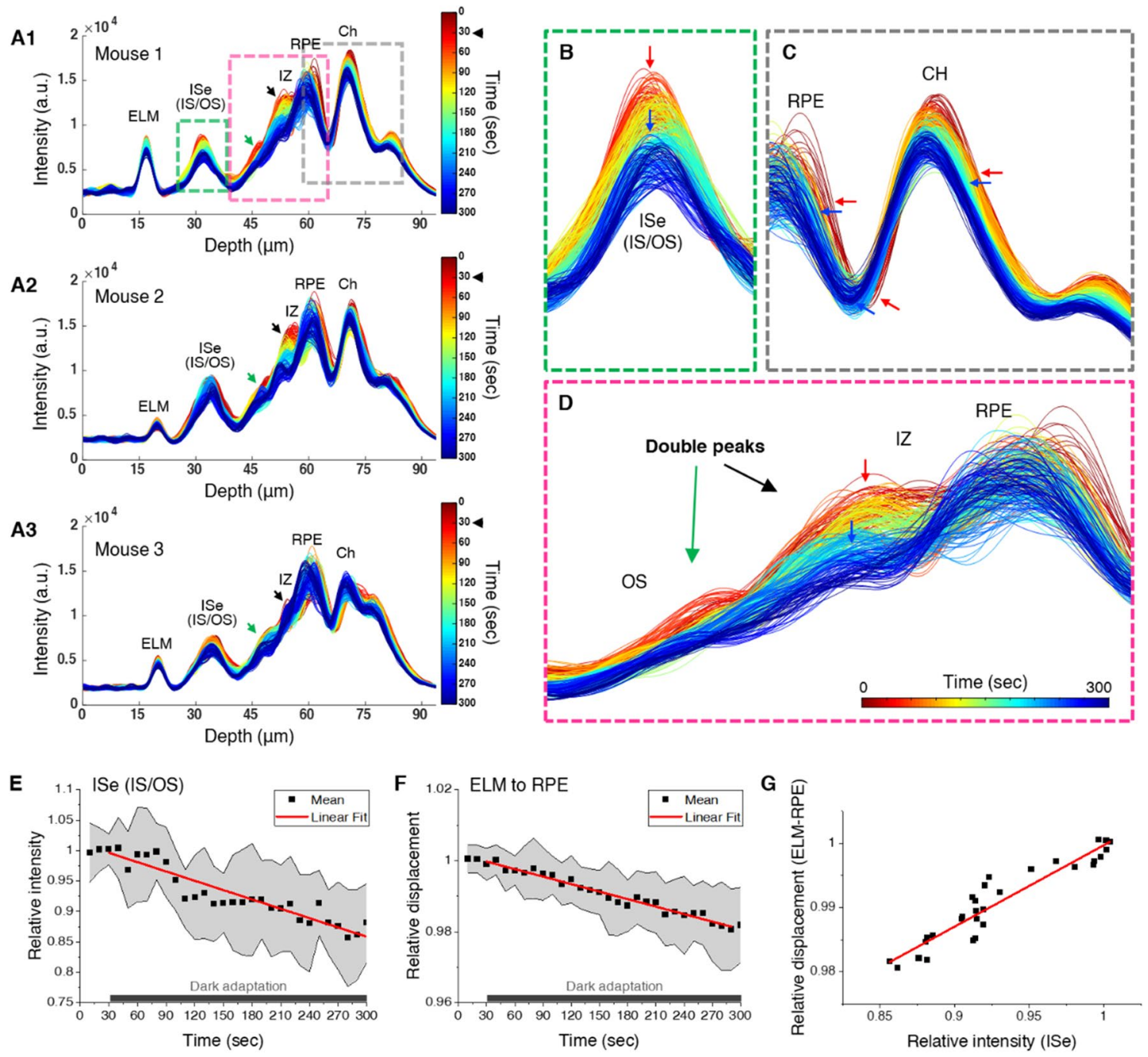

Figure 6. Quantitative analysis of high-speed OCT recording during transition to darkness. (A1-3) Colorcoded OCT intensity profiles of the outer retina from 3 different mice. Red and blue colors indicate a temporal gradient of a 30-min dark adaptation period. The lights were off at $30 \mathrm{~s}$. Green and black arrows indicate double peaks found along the photoreceptor OS. (B) Enlarged view of the ISe band, green box in (A1). (C) Enlarged view of the RPE and CH, grey box in (A1). (D) Enlarged view of the OS, IZ, and RPE, pink box in (A1). (E) Relative intensity changes and (F) ELM-RPE displacement during dark adaptation. Black dots represent the mean values, and the accompanied grey area represents standard deviation. (G) Correlation test between ISe intensity and ELM-RPE displacement. Black dots represent the mean values, and a red line is the linear fitting line. ELM: external limiting membrane; RPE: retinal pigment epithelium; ISe: inner segment ellipsoid; IS: inner segment; OS: outer segment; IZ: interdigitation zone; CH: Choroid. (A-D) were generated using MATLAB R2018b (http://mathwork.com), and (E-G) were generated using Origin 2020b (http://originlab.com).

to increased adenosine triphosphate (ATP) consumption to maintain the photoreceptor dark current ${ }^{6,39}$. This enhanced metabolism is mainly managed in the ISe, consisting of densely packed mitochondria. Due to complex internal geometry, mitochondria are the prominent light scatters in OCT imaging, and its geometrical configuration is actively modified based on metabolic demand through the fission and fusion process ${ }^{40}$. It is known that different mitochondrial configurations can alter the scattering property of light ${ }^{41,42}$, which may ultimately change the IOS property of the ISe. In line with this fact, fragmented mitochondria were frequently found in resting cells, while mitochondria became more fused when they were forced to rely on oxidative phosphorylation ${ }^{43}$. A recent study also demonstrated that mitochondria in the cone IS in hibernating ground squirrels were individually smaller and distorted, but mitochondria in active ground squirrels appeared elongated and well-organized. It was hypothesized that the elongated, parallel organization of mitochondria might enhance light delivery to the OS by reducing the number of refractive interfaces ${ }^{44}$. Taken together, it can be plausibly postulated that mitochondria in the ISe may undergo a fusion process during dark adaptation, forming tightly packed and elongated mitochondria to meet the high energy demand, resulting in low reflectance in the ISe band.

We also observed structural changes of the retina during dark adaptation. The mechanism of outer retinal thinning is rather well understood by RPE-mediated water removal from the ELM-RPE region. As discussed, the 

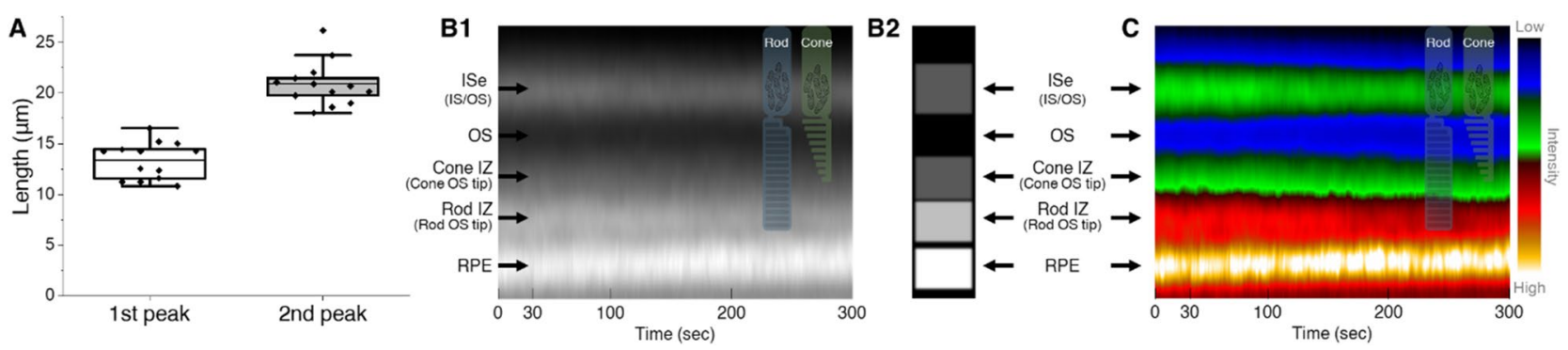

Figure 7. (A) Peak-to-peak length measurement from the ISe to double peaks along the photoreceptor OS $(\mathrm{N}=13)$. (B1) An average $\mathrm{M}$-scan image at the outer retinal region. (B2) A simplified intensity distribution model based on (B1). (C) OCT intensity in (B1) is depicted with a color-lookup-table "thal" provided in ImageJ software. The intensity range of the lookup table is shown in the bar to the right of the image. ISe inner segment ellipsoid, $I S$ inner segment, $O S$ outer segment, $I Z$ interdigitation zone, $R P E$ retinal pigment epithelium. (A) was generated using Origin 2020b (http://originlab.com). (B1-B2) were generated using Microsoft PowerPoint 2019 (http://microsoft.com). (C) was generated using ImageJ 1.52v (https://imagej.nih.gov/ij/).

transition from light to dark is accompanied by an increase in photoreceptor metabolism, resulting in increased oxygen consumption in the retina ${ }^{45}$. The increase in oxygen consumption leads to a proportionate increase in $\mathrm{CO}_{2}$ and wastewater production into the outer retina, which can acidify the outer retina and ultimately upregulate water removal co-transporters in the RPE. This rapid removal of the acidified water has been linked to significant thinning of the ELM-RPE region ${ }^{46,47}$. In fact, empirically derived acidic conditions by medicine and hypoxia were found to reduce the outer retinal volume directly ${ }^{48,49}$. Consistent with our results, Li et al. ${ }^{28}$ observed a significant reduction of outer retina thickness in the dark-adapted retina. They also reported that light-dependent volume changes in the outer retina varied with the stage of retinal degeneration in retinal degeneration 10 (rd10) mouse model ${ }^{50}$. Berkowitz et al. found strain-specific changes of the outer retina in different light conditions. A light-driven expansion of the outer retina was more prominent in C57BL/6 mice than 129S6/SvEvTac mice ${ }^{29}$. Gao et al. ${ }^{30}$ further demonstrated that dark adaptation significantly reduced the magnitude and width of a hypo-reflective band between the photoreceptor OS and RPE. In addition, Guziewicz et al. showed that the hypo-reflective band became more profound during the transition from dark to light in the canine BEST1 disease model, manifesting retinal abnormalities at the photoreceptor-RPE interface associated with defects in the RPE microvilli ensheathment ${ }^{51}$. Taken together, dynamic ORG measurement of dark adaptation can serve as a functional indicator for RPE-mediated water transport in the outer retina.

However, the water transport could not explain the altered integrity of the 3 rd outer retinal bands (i.e., IZ and OS tips). We instead hypothesize that interdigitating properties between the OS tip and RPE apical processes may be changed. In this study, we found double peaks along with the OS (Figs. 6A and 7), and these peaks became enervated during the dark adaptation period. This morphological configuration is quite similar to an axial positioning of cone and rod OS tips in the perifoveal outer retina in humans ${ }^{52-55}$. However, the putative cone IZ band has low reflectivity compared to the rod IZ band in the mouse retina, which is probably due to a sparse cone population. Considering the cone OS is shorter in length (Fig. 7A) and lower in occupancy than the rod OS in the mouse retina, the first and second peak most likely correspond to the cone and rod OS tip region, respectively. This ensheathing configuration has been also named as the contact cylinder ${ }^{53}$, where the RPE's long apical microvilli interdigitate with the photoreceptor OSs, supporting an adhesion between the retina and RPE. Microvilli are capable of active contraction while interdigitating with the $\mathrm{OS}^{51}$. It was demonstrated that the force required to detach the retina from the RPE was $20 \%$ greater in the light-adapted retina than in the dark-adapted retina $^{56}$. Also, retinal adhesion in wild-type mice increased by $58 \%$ after light adaptation ${ }^{57}$. To support the firm retinal attachment, tight ensheathment might be necessary for light adaptation, presumably manifesting high reflectance in the $3 \mathrm{rd}$ band. Conversely, the alleviated tension in darkness along with outer retinal thinning may loosen this interconnection, presumably manifesting low reflectance in the 3rd band. It should be noted that ensheathment of the OS by microvilli is not the only mechanism of retinal adhesion; interphotoreceptor matrix proteins simultaneously contribute to lowering retinal adhesive force in darkness ${ }^{5,58}$. In addition, either light modulated melanosome translocation ${ }^{59}$ or rearrangement between OS and microvilli60 during dark adaptation can also be a contributing factor in the $3 \mathrm{rd}$ IZ and OS tip band signal. In fact, the $3 \mathrm{rd}$ band is the most controversial band among researchers, and multiple interpretations for this region have been proposed ${ }^{54,61-63}$. Thus, the exact mechanism remains to be extensively investigated.

In conclusion, we demonstrated the feasibility of in vivo ORG assessment of dark adaptation to characterize dark-induced structural and reflectivity changes in the mouse retina. Compared to the conventional perimetry and ERG measurements, functional OCT enables intrinsic signal ORG dark adaptation kinetics in a quantitative way with layer-specificity. Moreover, the rapid testing protocol of dark adaptation kinetics would be suitable for both lab research and clinical application because of low subject burden. Although the complex correlations of homeostatic mechanisms and imaging features remain to be elucidated, the intrinsic signal ORG promises a great potential to enable noninvasive, objective measurement of dark adaptation kinetics, which can aid in the diagnosis of early-stage AMD, DR, and RP. 


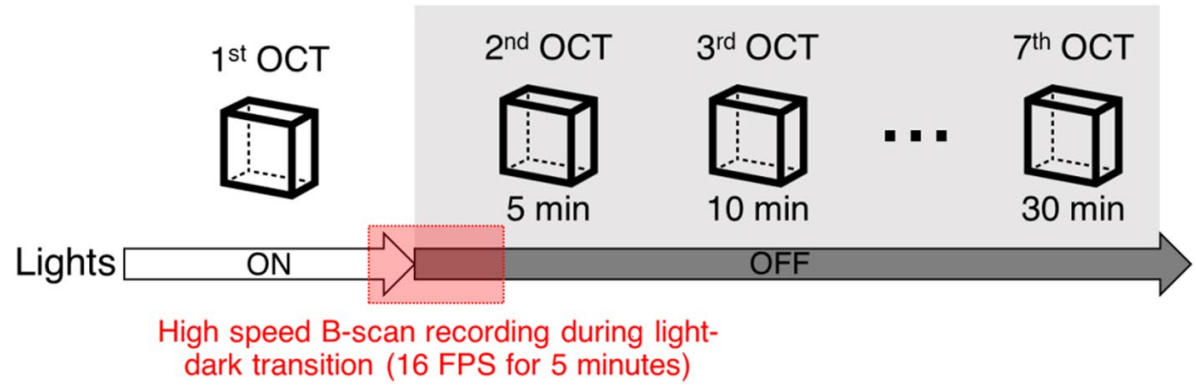

Figure 8. Image acquisition flow chart. The first OCT volume was acquired under ambient light conditions. After lights off, the second to seventh OCT volumes were sequentially acquired every $5 \mathrm{~min}$ up to $30 \mathrm{~min}$. In between the first and second OCT volume acquisition, high-speed B-scan recording was performed to monitor retinal response during a light-dark transitional moment.

\begin{abstract}
Methods
Animal preparation. Two-month-old C57BL/6 J mice of either sex (Jackson Laboratory, Bar Harbor, Maine, USA) were used in this study. The mice were kept in regular animal housing under a 14:10 h light/dark cycle. All animal experiments were approved by the local animal care and biosafety office and performed following the protocols approved by the Animal Care Committee (ACC) at the University of Illinois at Chicago. This study followed the Association for Research in Vision and Ophthalmology Statement for the Use of Animals in Ophthalmic and Vision Research, and we confirm that our work accords with the ARRIVE guidelines.
\end{abstract}

Imaging system. A custom-designed spectral-domain OCT system was used in this study. Technical details of the system are reported in our previous studies ${ }^{64,65}$. Briefly, a near-infrared super-luminescent diode $(\lambda=810 \mathrm{~nm} ; \Delta \lambda=100 \mathrm{~nm}$; D-810-HP, Superlum, Carrigtwohill, County Cork, Ireland) was used as the OCT light source. A line CCD camera with 2048 pixels (AViiVA EM4; e2v Technologies, Chelmsford, UK) was used in the custom-built OCT spectrometer. The axial and lateral resolutions of the system were theoretically estimated at 2.9 and $11 \mu \mathrm{m}$, respectively. The incident light power on the cornea was $1 \mathrm{~mW}$.

Experimental procedure. Imaging was performed in a laboratory room at $400 \mathrm{~lx}$. Stray light from electronic equipment was masked, and the imaging stage was shrouded with blackout fabric. Experiments were regularly performed between 12 and $3 \mathrm{pm}$. At the time of the experiment, the mouse retina was light-adapted due to constant ambient light conditions. The mouse was first anesthetized by intraperitoneal injection of a mixture of $100 \mathrm{mg} / \mathrm{kg}$ ketamine and $5 \mathrm{mg} / \mathrm{kg}$ xylazine. The anesthesia cocktail concentration was $10 \mathrm{mg} / \mathrm{mL}$ ketamine and $0.5 \mathrm{mg} / \mathrm{mL}$ xylazine. A drop of $1 \%$ tropicamide ophthalmic solution (Akorn, Lake Forest, IL) was applied to the imaging eye for pupil dilation, and a cover glass (12-545-80; Microscope cover glass, Fisherbrand, Waltham, MA) with a drop of eye gel (GenTeal, Novartis, Basel, Switzerland) was placed on the imaging eye. After the mouse was fully anesthetized, the head was fixed by a bite bar and ear bars in an animal holder. A heating pad was placed around the animal holder to keep the mouse warm. It took around 8-10 min between the time of intraperitoneal injection and the first OCT volume acquisition.

Image acquisition. Volumetric raster scans were performed for OCT imaging. Four repeated B-scans at each slow-scan position were collected and averaged to lower the speckle noise level; thus, each OCT volume consisted of $4 \times 600 \times 600 \mathrm{~A}$-scans over $1.2 \times 1.2 \mathrm{~mm}$ area. Seven OCT volumes were sequentially acquired with 5 min intervals at the same dorsal quadrant. Figure 8 illustrates the flow of image acquisition. The first volume was acquired in light condition, and the other six volumes were acquired in dark condition. High-speed recording to capture the initial moment of dark adaptation was conducted right after the first volume recording. The high-speed recording captured $4800 \mathrm{~B}$-scans at the same retinal plane with 16 frames per second rate for a complete 5-min recording. While recording, the room light was turned off at $30 \mathrm{~s}$ with the guidance of a LED blink synchronized with the OCT image acquisition system. Data acquisition and real-time imaging preview were made by a custom-built LabVIEW program (LabVIEW 2013, National Instruments, Austin, TX).

Image analysis. For quantitative analysis, retinal flattening was first implemented by realigning all A-lines in each OCT volume ${ }^{66}$. Next, adjacent 150 B-scans at the central region of OCT volume were averaged, followed by averaging 200 central A-lines, returning a single representative A-line per volume. The central region was carefully designated based on structural landmarks in each volume to secure regional consistency. Based on this A-line profile, retinal thickness and relative intensity value were measured. Each intensity profile was normalized based on the ONL intensity before relative intensity measurement in seven different OCT volumes. For the highspeed recording analysis, every $16 \mathrm{~B}$-scans were first averaged, followed by retinal flattening, which returned a total 300 B-scans, and each B-scan represents 1-s recording. 200 central A-lines were averaged for intensity and displacement measurement. To enhance the sampling density, each A-line was four times upsampled using linear interpolation. Image reconstruction and processing were done by MATLAB R2016a (MathWorks, Natick, MA). 
Statistical analysis. Data are expressed as mean \pm standard deviation unless otherwise indicated. Statistical analysis was carried out with one-way repeated measures ANOVA to compare OCT measurements between seven different time points, followed by post hoc tests using Bonferroni correction to detect significant differences between the time points. The $p$-values less than 0.05 were considered significant in all tests. For high-speed recording analysis, Pearson's correlation analysis was conducted to examine the strength of a linear relationship between OCT measurements at different time points. All statistical analyses were performed with Origin 2020b (OriginLab, Northampton, MA).

\section{Data availability}

Data supporting the findings of this manuscript are available from the corresponding author upon reasonable request.

Received: 2 September 2021; Accepted: 2 February 2022

Published online: 15 February 2022

\section{References}

1. Yang, G.-Q., Chen, T., Tao, Y. \& Zhang, Z.-M. Recent advances in the dark adaptation investigations. Int. J. Ophthalmol. 8, 12451252 (2015).

2. Lamb, T. D. \& Pugh, E. N. Jr. Phototransduction, dark adaptation, and rhodopsin regeneration the proctor lecture. Invest. Ophthalmol. Vis. Sci. 47, 5138-5152 (2006).

3. Kwan, C. C., Lee, H. E., Schwartz, G. \& Fawzi, A. A. Acute hyperglycemia reverses neurovascular coupling during dark to light adaptation in healthy subjects on optical coherence tomography angiography. Invest. Ophthalmol. Vis. Sci. 61, 38-38 (2020).

4. Calvert, P. D., Strissel, K. J., Schiesser, W. E., Pugh, E. N. Jr. \& Arshavsky, V. Y. Light-driven translocation of signaling proteins in vertebrate photoreceptors. Trends Cell Biol. 16, 560-568 (2006).

5. Ishikawa, M., Sawada, Y. \& Yoshitomi, T. Structure and function of the interphotoreceptor matrix surrounding retinal photoreceptor cells. Exp. Eye Res. 133, 3-18 (2015).

6. Linton, J. D. et al. Flow of energy in the outer retina in darkness and in light. Proc. Natl. Acad. Sci. 107, 8599 (2010).

7. Jackson, G. R. et al. Diagnostic sensitivity and specificity of dark adaptometry for detection of age-related macular degeneration. Invest. Ophthalmol. Vis. Sci. 55, 1427-1431 (2014).

8. Hsiao, C.-C., Hsu, H.-M., Yang, C.-M. \& Yang, C.-H. Correlation of retinal vascular perfusion density with dark adaptation in diabetic retinopathy. Graefes Arch. Clin. Exp. Ophthalmol. 257, 1401-1410 (2019).

9. Herse, P. Retinitis pigmentosa: Visual function and multidisciplinary management. Clin. Exp. Optom. 88, 335-350 (2005).

10. Group A-REDSR. A randomized, placebo-controlled, clinical trial of high-dose supplementation with vitamins $C$ and $E$, beta carotene, and zinc for age-related macular degeneration and vision loss: AREDS report no. 8. Arch Ophthalmol (Chicago, Ill : 1960) 119, 1417-1436 (2001).

11. Group A-REDSR. Lutein + zeaxanthin and omega-3 fatty acids for age-related macular degeneration: The age-related eye disease study 2 (AREDS2) randomized clinical trial. Jama 309, 2005-2015 (2013).

12. Suppiej, A. et al. Early onset retinal dystrophies: Clinical clues to diagnosis for pediatricians. Ital. J. Pediatr. 45, 168 (2019).

13. Early Treatment Diabetic Retinopathy Study Research Group. Grading diabetic retinopathy from stereoscopic color fundus photographs: An extension of the modified airlie house classification: ETDRS report number 10. Ophthalmology 98, 786-806 (1991).

14. Mahroo, O. A. R. \& Lamb, T. D. Recovery of the human photopic electroretinogram after bleaching exposures: Estimation of pigment regeneration kinetics. J. Physiol. 554, 417-437 (2004).

15. Azimipour, M. et al. Optoretinogram: Optical measurement of human cone and rod photoreceptor responses to light. Opt. Lett. 45, 4658-4661 (2020).

16. Cooper, R. F., Brainard, D. H. \& Morgan, J. I. W. Optoretinography of individual human cone photoreceptors. Opt. Express 28, 39326-39339 (2020).

17. Pandiyan, V. P. et al. The optoretinogram reveals the primary steps of phototransduction in the living human eye. Sci. Adv. 6, eabc1 $124(2020)$

18. Son, T., Kim, T.-H., Ma, G., Kim, H. \& Yao, X. Functional intrinsic optical signal imaging for objective optoretinography of human photoreceptors. Exp. Biol. Med. 246, 639-643 (2020).

19. Zhang, P. et al. Measurement of diurnal variation in rod outer segment length in vivo in mice with the OCT optoretinogram. Invest. Ophthalmol. Vis. Sci. 61, 9-9 (2020).

20. Lassoued, A. et al. Cone photoreceptor dysfunction in retinitis pigmentosa revealed by optoretinography. Proc. Natl. Acad. Sci. 118, e2107444118 (2021).

21. Hillmann, D. et al. In vivo optical imaging of physiological responses to photostimulation in human photoreceptors. Proc. Natl. Acad. Sci. 113, 13138 (2016).

22. Ma, G., Son, T., Kim, T.-H. \& Yao, X. In vivo optoretinography of phototransduction activation and energy metabolism in retinal photoreceptors. J. Biophotonics 14, e202000462 (2021).

23. Hunter, J. J., Merigan, W. H. \& Schallek, J. B. Imaging retinal activity in the living eye. Annu. Rev. Vision Sci. 5, 15-45 (2019).

24. Zhang, L., Dong, R., Zawadzki, R. J. \& Zhang, P. Volumetric data analysis enabled spatially resolved optoretinogram to measure the functional signals in the living retina. J. Biophotonics n/a, e202100252 (2021).

25. Messner, A. et al. Quantification of intrinsic optical signals in the outer human retina using optical coherence tomography. Ann. N. Y. Acad. Sci. 1-13 (2021).

26. Yao, X. C., Yamauchi, A., Perry, B. \& George, J. S. Rapid optical coherence tomography and recording functional scattering changes from activated frog retina. Appl. Opt. 44, 2019-2023 (2005).

27. Kim, T.-H., Wang, B., Lu, Y., Son, T. \& Yao, X. Functional optical coherence tomography enables in vivo optoretinography of photoreceptor dysfunction due to retinal degeneration. Biomed. Opt. Express 11, 5306-5320 (2020).

28. Li, Y., Fariss, R. N., Qian, J. W., Cohen, E. D. \& Qian, H. Light-induced thickening of photoreceptor outer segment layer detected by ultra-high resolution OCT imaging. Invest. Ophthalmol. V. Sci. OCT57, OCT105-111 (2016).

29. Berkowitz, B. A. et al. Mitochondrial respiration in outer retina contributes to light-evoked increase in hydration in vivo. Invest. Ophthalmol. Vis. Sci. 59, 5957-5964 (2018).

30. Gao, S. et al. Functional regulation of an outer retina hyporeflective band on optical coherence tomography images. Sci. Rep. 11, 10260 (2021).

31. Lu, C. D. et al. Photoreceptor layer thickness changes during dark adaptation observed with ultrahigh-resolution optical coherence tomography. Invest. Ophthalmol. Vis. Sci. 58, 4632-4643 (2017).

32. Yao, X., Son, T., Kim, T.-H. \& Lu, Y. Functional optical coherence tomography of retinal photoreceptors. Exp. Biol. Med. 243, 1256-1264 (2018). 
33. Yao, X. \& Kim, T.-H. Fast intrinsic optical signal correlates with activation phase of phototransduction in retinal photoreceptors. Exp. Biol. Med. 245, 1087-1095 (2020).

34. Eric, M. M. et al. Evaluating anesthetic protocols for functional blood flow imaging in the rat eye. J. Biomed. Opt. 22, 1-6 (2017).

35. Janssen, B. J. A. et al. Effects of anesthetics on systemic hemodynamics in mice. Am. J. Physiol.-Heart Circ. Physiol. 287, H1618H1624 (2004).

36. Fu, Y. \& Yau, K.-W. Phototransduction in mouse rods and cones. Pflug. Arch. 454, 805-819 (2007).

37. Zhang, P. et al. In vivo optophysiology reveals that G-protein activation triggers osmotic swelling and increased light scattering of rod photoreceptors. Proc. Natl. Acad. Sci. 114, E2937 (2017).

38. Lee, K. E., Heitkotter, H. \& Carroll, J. Challenges associated with ellipsoid zone intensity measurements using optical coherence tomography. Trans. Vision Sci. Technol. 10, 27-27 (2021).

39. Ingram, N. T., Fain, G. L. \& Sampath, A. P. Elevated energy requirement of cone photoreceptors. Proc. Natl. Acad. Sci. 117, 19599 (2020).

40. Youle, R. J. \& van der Bliek, A. M. Mitochondrial fission, fusion, and stress. Science 337, 1062 (2012).

41. Haseda, K. et al. Significant correlation between refractive index and activity of mitochondria: single mitochondrion study. Biomed. Opt. Express 6, 859-869 (2015).

42. Tychinsky, V. The metabolic component of cellular refractivity and its importance for optical cytometry. J. Biophotonics 2, 494-504 (2009).

43. Westermann, B. Bioenergetic role of mitochondrial fusion and fission. Biochim. et Biophys. Acta (BBA): Bioenerg. 1817, 1833-1838 (2012).

44. Ball, J. M., Chen, S. \& Li, W. Cone mitochondria act as microlenses to enhance light delivery and confer Stiles-Crawford-like direction sensitivity. bioRxiv, https://doi.org/10.1101/2021.02.11.430818 (2021).

45. Adijanto, J., Banzon, T., Jalickee, S., Wang, N. S. \& Miller, S. S. CO2-induced ion and fluid transport in human retinal pigment epithelium. J. Gen. Physiol. 133, 603-622 (2009).

46. Wangsa-Wirawan, N. D. \& Linsenmeier, R. A. Retinal oxygen: Fundamental and clinical aspects. Arch. Ophthalmol. 121, 547-557 (2003).

47. Berkowitz, B. A. \& Qian, H. OCT imaging of rod mitochondrial respiration in vivo. Exp. Biol. Med. 246, 15353702211013800 (2021).

48. Wolfensberger, T. J., Dmitriev, A. V. \& Govardovskii, V. I. Inhibition of membrane-bound carbonic anhydrase decreases subretinal pH and volume. Doc. Ophthalmol. 97, 261-271 (1999).

49. Cao, W., Govardovskii, V., Li, J. D. \& Steinberg, R. H. Systemic hypoxia dehydrates the space surrounding photoreceptors in the cat retina. Invest. Ophthalmol. Vis. Sci. 37, 586-596 (1996).

50. Li, Y. et al. Light-dependent OCT structure changes in photoreceptor degenerative rd 10 Mouse Retina. Invest. Ophthalmol. Vis. Sci. 59, 1084-1094 (2018).

51. Guziewicz, K. E. et al. BEST1 gene therapy corrects a diffuse retina-wide microdetachment modulated by light exposure. Proc. Natl. Acad. Sci. 115, E2839 (2018).

52. Liu, Z., Kocaoglu, O. P. \& Miller, D. T. 3D imaging of retinal pigment epithelial cells in the living human retina. Invest. Ophthalmol. V. Sci. 57, OCT533-OCT543 (2016).

53. Spaide, R. F. \& Curcio, C. A. Anatomical correlates to the bands seen in the outer retina by optical coherence tomography: Literature review and model. Retina 31, 1609 (2011).

54. Yao, X., Son, T., Kim, T.-H. \& Le, D. Interpretation of anatomic correlates of outer retinal bands in optical coherence tomography. Exp. Biol. Med. 246, 15353702211022674 (2021).

55. Chong, S. P. et al. Ultrahigh resolution retinal imaging by visible light OCT with longitudinal achromatization. Biomed. Opt. Express 9, 1477-1491 (2018).

56. Owczarek, F. R., Marak, G. E. \& Pilkerton, A. R. Retinal adhesion in light- and dark-adapted rabbits. Invest. Ophthalmol. Vis. Sci. 14, 353-358 (1975).

57. Nandrot, E. F., Anand, M., Sircar, M. \& Finnemann, S. C. Novel role for av $\beta 5$-integrin in retinal adhesion and its diurnal peak. Am. J. Physiol. Cell Physiol. 290, C1256-C1262 (2006).

58. Marmor, M. F. Chapter 8 mechanisms of retinal adhesion. Progr. Retinal Res. 12, 179-204 (1993).

59. Zhang, Q.-X. et al. In vivo optical coherence tomography of light-driven melanosome translocation in retinal pigment epithelium. Sci. Rep. 3, 2644 (2013).

60. Kocaoglu, O. P. et al. Photoreceptor disc shedding in the living human eye. Biomed. Opt. Express 7, 4554-4568 (2016).

61. Bloom, S. M. \& Singal, I. P. revised classification of the optical coherence tomography outer retinal bands based on central serous chorioretinopathy analysis. Retina 41, 181-188 (2021).

62. Cuenca, N. et al. Interpretation of OCT and OCTA images from a histological approach: Clinical and experimental implications. Progr. Retinal Eye Res. 77, 100828 (2020).

63. Jonnal, R. S. et al. The cellular origins of the outer retinal bands in optical coherence tomography images. Invest. Ophthalmol. Vis. Sci. 55, 7904-7918 (2014).

64. Son, T. et al. Optical coherence tomography angiography of stimulus evoked hemodynamic responses in individual retinal layers. Biomed. Opt. Express 7, 3151-3162 (2016).

65. Kim, T.-H., Son, T., Klatt, D. \& Yao, X. Concurrent OCT and OCT angiography of retinal neurovascular degeneration in the 5XFAD Alzheimer's disease mice. Neurophotonics 8, 1-12 (2021).

66. Kim, T.-H., Son, T., Lu, Y., Alam, M. \& Yao, X. comparative optical coherence tomography angiography of wild-type and rd10 mouse retinas. Trans. V. Sci. Technol. 7, 42-42 (2018).

\section{Acknowledgements}

This research was supported in part by the National Institutes of Health (NIH) Grants R01EY023522, R01EY030101, R01EY030842, R01EY029673, and P30EY001792; by the Chicago Biomedical Consortium with support from the Searle Funds at the Chicago Community Trust; by the Richard and Loan Hill endowment; and by an unrestricted grant from Research to Prevent Blindness.

\section{Author contributions}

T.K. contributed to data collection, data processing, data analysis, and manuscript preparation; J.D. contributed to data analysis. X.Y. supervised the project and contributed to the study design, data analysis, and manuscript preparation.

\section{Competing interests}

The authors declare no competing interests. 


\section{Additional information}

Correspondence and requests for materials should be addressed to X.Y.

Reprints and permissions information is available at www.nature.com/reprints.

Publisher's note Springer Nature remains neutral with regard to jurisdictional claims in published maps and institutional affiliations.

(c) (1) Open Access This article is licensed under a Creative Commons Attribution 4.0 International License, which permits use, sharing, adaptation, distribution and reproduction in any medium or format, as long as you give appropriate credit to the original author(s) and the source, provide a link to the Creative Commons licence, and indicate if changes were made. The images or other third party material in this article are included in the article's Creative Commons licence, unless indicated otherwise in a credit line to the material. If material is not included in the article's Creative Commons licence and your intended use is not permitted by statutory regulation or exceeds the permitted use, you will need to obtain permission directly from the copyright holder. To view a copy of this licence, visit http://creativecommons.org/licenses/by/4.0/.

(C) The Author(s) 2022 